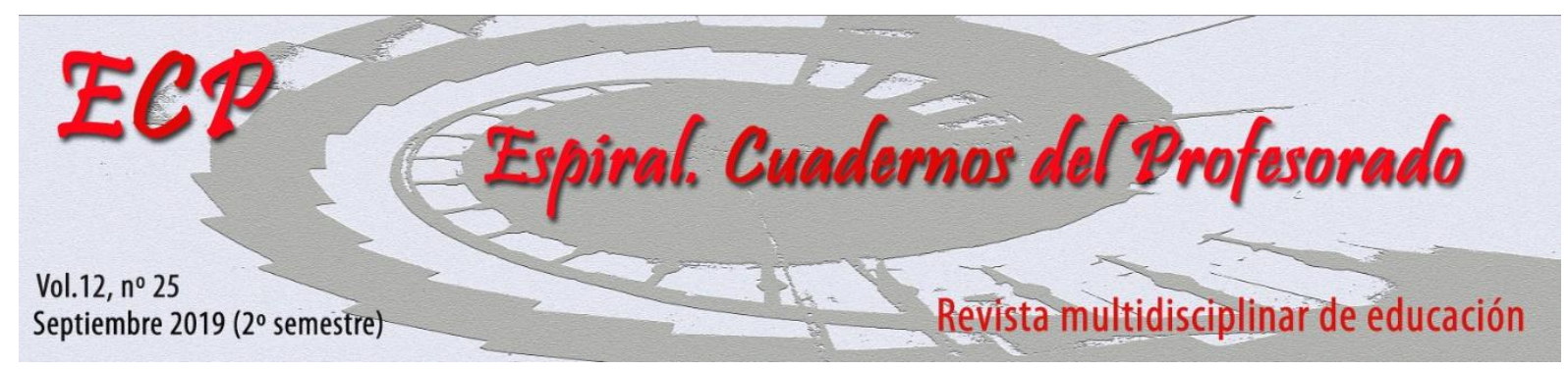

\title{
Protocolo de estudio de las variables académicas, psicológicas y de actividad física que influyen en el rendimiento académico de adolescentes mexicanos y españoles
}

\section{Protocol for the study of the academic, psychological and physical activity variables that influence the academic performance of Mexican and Spanish adolescents}

\author{
Raul Baños ${ }^{1}$, Michelle Barretos-Ruvalcaba ${ }^{1}$, y Antonio Baena-Extremera ${ }^{2}$ \\ ${ }^{1}$ Universidad Autónoma de Baja California, México; ${ }^{2}$ Universidad de Granada, España
}

\section{Resumen}

El objetivo del presente trabajo será analizar las diferencias académicas, personales y ambientales de los estudiantes pertenecientes a los estados de Baja California y Nuevo León (México) y de la Comunidad Autónoma (CCAA) de Andalucía (España). El diseño de la muestra será probabilístico por centros, estratificado, polietápico, por afijación proporcional, teniendo en cuenta que se seleccionará la muestra de dos Estados de México (Nuevo León y Baja California) y la Comunidad Autónoma de Andalucía (España). Los centros de educación secundaria que participarán en el proyecto de investigación serán seleccionados de forma aleatoria simple. El instrumento a utilizar será un cuestionario compuesto por las escalas: Test de Matrices Progresivas de Raven, Rendimiento académico, Trait Meta Mood Scale-24 (TMMS-24), Escala de Autoestima Global de Rosenberg (EAG), Physical Education Classroom Instrument (PECI), Satisfacción con la vida (SATV), Satisfacción con la escuela (SATD), Satisfacción Intrínseca con las materias (SIM), Niveles de actividad física en el tiempo libre. En este artículo se presenta el diseño de la investigación. Pensamos que los hallazgos de la presente investigación tendrán una gran repercusión en los sistemas educativos de ambos países. Los hallazgos del estudio se difundirán a través de revistas de impacto científico y presentaciones en conferencias de carácter internacional.

Palabras clave: Educación secundaria; rendimiento académico; adolescentes; coeficiente intelectual; actividad física.

\begin{abstract}
The objective of the present work will be to analyze the academic, personal and environmental differences of the students belonging to the states of Baja California and Nuevo León (Mexico) and of the Autonomous Community (CCAA) of Andalucía (Spain). The sample design will be probabilistic by centers, stratified, multi-stage, by proportional affixation, taking into account that the sample will be selected from two States of Mexico (Nuevo León and Baja California) and the Autonomous Community of Andalucía (Spain). Secondary education centers that will participate in the research project will be selected in a simple random manner. The instrument to be used will be a questionnaire composed of scales: Raven Progressive Matrices Test, Academic Performance, Trait Meta Mood Scale-24 (TMMS-24), Rosenberg Global Self-Esteem Scale (EAG), Physical Education Classroom Instrument (PECI), Satisfaction with life (SATV), Satisfaction with school (SATD), Intrinsic satisfaction with the subjects (SIM) and Levels of physical activity in free time. In this article we present the design of the research. We believe that the findings of this research will have a great impact on the educational systems of both countries. The findings of the study will be disseminated through scientific impact journals and presentations at international conferences.
\end{abstract}

Keywords: Secondary school; academic performance; adolescents; intelligence quotient; physical activity.

Fecha de recepción: 21/05/2019

Fecha de aceptación: 24/07/2019

Correspondencia: Antonio Baena-Extremera, Universidad de Granada (España); E-mail: abaenaextrem@ugr.es 
Protocolo de estudio de las variables académicas, psicológicas y de actividad física que influyen en el rendimiento académico de adolescentes mexicanos y españoles

\section{Introduccion}

El bajo rendimiento académico en los adolescentes mexicanos es un hecho más que patente si observamos los últimos resultados del Informe Pisa (2015), planteándose así un serio problema en el sistema educativo de México. Si comparamos los resultados obtenidos por los estudiantes mexicanos con el de promedio de los países de la Organización para la Cooperación y el Desarrollo Económico (OCDE, 2016), la preocupación aumenta, ya que menos del 1\% de los estudiantes en México logran alcanzar los niveles mínimos de competencia de excelencia en las tres áreas que evalúa el Informe Pisa (matemáticas, ciencia y lectura).

La literatura científica ha manifestado que diversas variables se han relacionado con el rendimiento académico en los últimos años, por ejemplo, Baños, Ortiz-Camacho, Baena-Extremera y Tristán-Rodríguez (2017), proponen analizar el rendimiento académico desde el bienestar subjetivo de los estudiantes. Además, otros estudios han demostrado que las calificaciones de los estudiantes se relacionan con los comportamientos disruptivos en el aula (Collins et al., 2016), la inteligencia emocional (Usán \& Salavera, 2019), inteligencia cognitiva (Gómez-Veiga, Vila, Duque, \& García, 2018), los niveles de actividad física (Wassenaar, 2019) y la autoestima (Díaz \& Caso, 2018).

La teoría del bienestar subjetivo de Diener (2009), estudia el nivel de satisfacción de las personas, niveles bajos y preocupantes en adolescentes mexicanos (OCDE, 2016). Esta teoría, estudia como los individuos realizan juicios evaluativos sobre su satisfacción con la vida o con áreas específicas de la vida (dimensión cognitiva), y sobre cómo identifican las emociones (afectos positivos y negativos) como la diversión y el aburrimiento (Diener, 2009). De esta forma, para que un estudiante consiga el éxito académico es necesario que se sienta a gusto y satisfecho con la escuela en general (Koroboca \& Starobin, 2015), y de forma más específica con cada una de las materias que componen su proceso de enseñanza y aprendizaje (Baños, Baena-Extremera \& Ortiz-Camacho, en prensa). Además, el bienestar subjetivo de los adolescentes también se relaciona con el índice de masa corporal (IMC), que este a su vez, se relaciona con el autocontrol, la sociabilidad y la inteligencia emocional (Ruiz-Ariza, SuárezManzano, López-Serrano, \& Martínez-López, 2019). Por el contrario, la insatisfacción con la escuela se relaciona con un bajo rendimiento académico (Pekrun, Goetz, Daniels, Stupnisky \& Perry, 2010), debilitando la eficiencia de cualquier metodología de aprendizaje (Ahmed, Van der Werf, Kuyper \& Minnaert, 2013), siendo crucial que el docente desarrolle unas buenas competencias para mejorar la satisfacción de sus estudiantes (Invernizzi et al., 2019). Sin embargo, si analizamos los bajos resultados académicos, el bienestar subjetivo y desempeño académico del Informe Pisa (2015), se puede identificar la gran problemática que se enfrentan los estudiantes de secundaria mexicanos.

La inteligencia emocional es otro factor que ha demostrado una gran repercusión en el desarrollo de los adolescentes. Concretamente, Mayer y Salovey (1997, p. 10), identifican varias características de las personas emocionalmente inteligentes: a) la capacidad de percibir con precisión, evaluar y expresar emociones; b) la capacidad de acceder y/o generar sentimientos que faciliten el pensamiento; c) capacidad de entender la emoción y el conocimiento emocional; y d) la capacidad de regular las emociones para promover el crecimiento emocional e intelectual. La literatura científica ha evidenciado que la actividad física, la danza y participar en actividades físico-creativas como obras teatrales, aumentan los niveles de inteligencia emocional (González, Cayuela, \& López, 2019) y el autoestima (Umstattd, Bridges, Schmid, Hecht, \& Pollack, 2019). Además, la actividad física ayuda a mejorar el rendimiento cognitivo a través de dos mecanismos, por un lado, la capacidad cardiorrespiratoria induce angiogénesis en la corteza motora incrementando el flujo sanguíneo, mejorando así la vascularización cerebral, por otro, incrementa los niveles del factor neurotrófico derivado del cerebro, aumentando la supervivencia neuronal (Adkins, Boychuk, Remple, \& Kleim, 2006). Sin embargo, los niveles de actividad física en el tiempo libre de los adolescentes mexicanos son verdaderamente preocupantes, como ya apuntan Ruiz-Juan, Baena-Extremera y Baños (2017).

Otra variable que incide sobre el contexto de los adolescentes es la autoestima. En función de cómo se diviertan y se sientan satisfechos los estudiantes con la escuela, podrán incrementar sus niveles de autoestima (Baena-Extremera \& Granero-Gallegos, 2013), mejorando también el desempeño académico, sintiéndose mejor consigo mismos y con su propia vida (Góngora, \& Casullo, 2009). 
También, el bajo autoconcepto de los adolescentes se relaciona con mayores niveles de comportamientos disruptivos en la escuela (Lewis et al., 2013), causando un impacto negativo en el entorno de aprendizaje (Kulinna, Cothran, \& Regualos, 2006). En esta línea, se ha demostrado que el aburrimiento en la escuela repercute positivamente en mayores niveles de conductas disruptivas y en la falta de apego a las instituciones sociales (Feinberg, Sakuma, Hostetler, \& Mc Hale, 2013). Las consecuencias del aburrimiento en la escuela, no se relacionan únicamente con comportamientos negativos en el aula, sino que pueden generar consecuencias más graves en cuanto a conductas de alto riesgo, como el beber, consumo de drogas, carreras de automóviles y acciones delictivas (Newberry \& Duncan, 2001; Wegner \& Flisher, 2009; Yang \& Yoh 2005), todo ello repercutiendo sobre el rendimiento académico de los estudiantes.

La preocupación sobre el rendimiento académico de los adolescentes mexicanos se hace más latente, cuando se observa que no ha habido mejoras significativas desde el 2003 (OCDE, 2016). Sin embargo, sus homólogos españoles de habla castellana si han ido mejorando los resultados de dichas evaluaciones. Por esto, creemos interesante en indagar sobre cómo repercuten las variables anteriormente descritas en el rendimiento con la escuela de los estudiantes mexicanos.

Acorde con lo mencionado en los anteriores párrafos y tras la problemática del bajo rendimiento académico en los adolescentes mexicanos, en comparación con los estudiantes españoles que hablan el mismo idioma, nos planteamos como preguntas las siguientes: ¿existirán diferencias en las variables académicas, personales y ambientales, entre estudiantes españoles y mexicanos?, ¿obtendrán los mismos niveles de satisfacción con las materias de matemáticas, español y educación física los estudiantes de ambos países?, ¿tendrán los estudiantes españoles mayores niveles de inteligencia emocional, cognitiva y de actividad física que los mexicanos, influenciando así en el rendimiento académico?, ¿qué adolescentes tendrán mayores niveles de comportamientos negativos?. Teniendo en cuenta estas cuestiones, consideramos que sería de gran interés para la comunidad educativa y científica responder a las preguntas anteriormente mencionadas. Para ello, describimos a continuación el diseño del futuro trabajo de investigación.

\section{Método}

\section{Hipótesis y objetivos del estudio}

A continuación, se describirán las hipótesis $(\mathrm{H})$ y objetivos $(\mathrm{O})$ general y específico del trabajo que se pretende desarrollar:

- $H_{\text {general: }}$ Los estudiantes españoles obtendrán valores medios más altos en las variables que se relacionen con un mejor rendimiento académico y los estudiantes mexicanos obtendrán valores medios más altos en las variables que se relacionen con un menor rendimiento académico.

- $O_{\text {general: }}$ Analizar las diferencias académicas, personales y ambientales de los estudiantes pertenecientes a los estados de Baja California y Nuevo León (México) y de la Comunidad Autónoma (CCAA) de Andalucía (España).

- $H_{1:}$ Los estudiantes con mejor rendimiento académico se relacionarán con niveles más bajos de IMC y más altos de satisfacción con la vida, satisfacción con las materias de EF, Matemáticas y Lengua.

- $O_{1:}$ Analizar los perfiles de los estudiantes en función del rendimiento académico, el IMC, la satisfacción con la vida y satisfacción con las materias de EF, Matemáticas y Lengua.

- $H_{2}$ La inteligencia emocional predecirá positivamente los niveles de actividad física en su tiempo libre y éste a su vez el coeficiente intelectual.

- $\mathrm{O}_{2 \text { : }}$ Conocer las relaciones de predicción entre la inteligencia emocional, los niveles de actividad física en su tiempo libre y el coeficiente intelectual de los estudiantes. 
- $H_{3:}$ Un nivel de autoestima alto de los estudiantes predecirá de forma negativa los comportamientos negativos en el aula de EF y éstos a su vez la satisfacción con la escuela.

- $O_{3:}$ Analizar como la autoestima de los adolescentes predice los comportamientos negativos en el aula de EF y éstos a su vez la satisfacción con la escuela.

\section{Diseño}

El diseño del trabajo es no experimental, seccional, descriptivo y predictivo. El trabajo no es experimental debido a que no se tratará a ningún grupo con ningún tipo de intervención, para comprobar sus efectos en los sujetos analizados. Es seccional, porque se tomará una muestra en un momento concreto, sin ser longitudinal ni realizar varias tomas de datos. Será descriptivo, porque analizaremos de forma descriptiva las variables seleccionadas en cada uno de los instrumentos seleccionados en el apartado correspondiente. Finalmente será un estudio predictivo, pues usaremos análisis de regresión lineal multivariado y modelos de ecuaciones estructurales, para responder a los objetivos previstos en el apartado correspondiente.

\section{Muestra}

El diseño de la muestra será probabilístico por centros, estratificado, polietápico, por afijación proporcional, teniendo en cuenta que se seleccionará la muestra de dos Estados de México (Nuevo León y Baja California) y la Comunidad Autónoma de Andalucía (España). Los centros de educación secundaria que participarán en el proyecto de investigación serán seleccionados de forma aleatoria simple.

Para ello se tendrán en cuenta los datos reportados por la SEP en México y el Ministerio Educación, Cultura y Deporte y las Delegaciones Provinciales de con competencias en educación en España. De esta manera, se podrá hacer comparaciones representativas entre los Estados de México, la CCAA en España y a su vez, entre países.

En la Tabla 1, se puede observar el número de estudiantes de tercer nivel de educación secundaria en los estados de México y la CCAA de Andalucía, España, la muestra representativa indicada en función del sexo y del estado, para una población finita con un nivel de confianza del 95\% y para un margen de error de $\pm 5 \%$.

Tabla 1.

Universo y muestra representativa dividas por estados y sexo.

\begin{tabular}{cccc}
\hline & & Chicas & Chicos \\
\hline \multirow{2}{*}{ Nuevo León } & Universo & 13396 & 13831 \\
& Muestra & 374 & 374 \\
\multirow{2}{*}{ Baja California } & Universo & 13176 & 13627 \\
& Muestra & 374 & 374 \\
Andalucía & Universo & 33787 & 34327 \\
& Muestra & 380 & 380 \\
\hline
\end{tabular}

\section{Instrumentos}

Test de Matrices Progresivas de Raven. Para llevar a cabo esta investigación, primero se medirá la capacidad cognitiva no verbal de los adolescentes con el Test de Matrices Progresivas de Raven (Raven, Styles \& Raven, 1998), una evolución del TMPR original (Raven, 1938). El TMPR presenta a los participantes 60 rompecabezas visuales en 5 series de 12. Cada rompecabezas está formado por una matriz (generalmente $2 \times 2$ o $3 \times 3$ ) que muestra el cambio a lo largo de los ejes x e y. Falta una pieza de cada matriz y debe identificarse por selección múltiple entre seis opciones (conjuntos A-B) u ocho opciones (conjuntos C-E). La dificultad aumenta progresivamente dentro de cada conjunto y de un conjunto a otro. Las puntuaciones van desde 0-60. El TMPR tiene una buena confiabilidad de prueba y reexpresión durante períodos de hasta un año en una variedad de culturas, y muy buena validez concurrente y de construcción (Raven, 2000). Para realizar esta prueba los estudiantes dispondrán de un tiempo máximo de 60 minutos. 
Rendimiento académico. Para evaluar el rendimiento académico de los estudiantes, se solicitará a los docentes del centro educativo las últimas calificaciones obtenidas en la última evaluación, siguiendo el procedimiento empleado por otros estudios (Muhonen, Pakarinen, Poikkeus, Lerkkanen, \& Rasku-Puttonen, 2018; Ramos, López-Fernández, \& Llamas-Salguero, 2017). Las respuestas se recogerán con una escala tipo Likert desde el 1 (la calificación más baja), hasta 10 (la calificación más alta), tal como se evalúa a los estudiantes.

Trait Meta Mood Scale-24 (TMMS-24). Para medir la inteligencia emocional en los adolescentes se utilizará la versión española del TMMS-24 de Salguero, Fernández-Berrocal, Balluerka, y Aritzeta (2010), de la versión en inglés desarrollado por Salovey, Mayer, Goldman, Turvey, y Palfai (1995). Este instrumento presenta 24 ítems que miden el grado de inteligencia emocional de los adolescentes en tres dimensiones: atención emocional, claridad de sentimientos y reparación emocional. Cada factor está compuesto por ocho ítems. La escala está precedida por la frase: "Dinos tu grado de desacuerdo o acuerdo...". Un ejemplo de ítem de atención emocional es "Presto mucha atención a los sentimientos", un ejemplo de ítem de claridad emocional es "Frecuentemente puedo definir mis sentimientos" y un ejemplo de ítem de reparación de las emociones es "Aunque a veces me siento triste, suelo tener una versión optimista". Las respuestas se recogerán en una escala de tipo Likert de 5 puntos que oscila desde 1 (muy en desacuerdo) a 5 (muy de acuerdo).

Escala de Autoestima Global de Rosenberg (EAG). Para medir la autoestima, se usará el Test de Autoestima de Rosenberg (1965), adaptado al castellano por Atienza, Moreno y Balaguer (2000). Este instrumento presenta 10 ítems, cinco redactados de forma positiva y cinco de forma negativa, que miden el grado de autoestima global. La escala está precedida por la frase: "Dinos tu grado de desacuerdo o acuerdo...". Un ejemplo de ítem es "A veces pienso que no sirvo para nada". Las respuestas se recogerán en una escala de tipo Likert de 4 puntos que oscila desde 1 (muy en desacuerdo) a 5 (muy de acuerdo).

Physical Education Classroom Instrument (PECI). Se utilizará la versión de Granero-Gallegos y Baena-Extremera (2016) adaptado a Educación Física. Este instrumento está compuesto por 20 ítems que miden las percepciones que tienen los estudiantes sobre su conducta en el aula de EF. Está compuesto por cinco dimensiones: agresividad (4 ítems), irresponsabilidad y bajo compromiso (4 ítems), desobediencia de las normas (4 ítems), perturbador del ambiente de clase (4 ítems) y bajo autocontrol personal (4 ítems). La escala está precedida por la frase "Piensa en tu propio comportamiento en clase de Educación Física y dinos tu grado de desacuerdo o acuerdo...”. Un ejemplo de ítem de agresividad es "Hablo de forma correcta al profesor/a", de irresponsabilidad y bajo compromiso "Busco llamar la atención", de desobediencia de las normas "No sigo las instrucciones", de perturbador del ambiente de clase "Miento en las clases de EF", y de bajo autocontrol personal "Soy peleón". Las respuestas se recogerán en escala tipo Likert desde 1 (muy en desacuerdo) hasta 5 (muy de acuerdo).

Satisfacción con la vida (SATV). Para medir la satisfacción con la vida, se utilizará la escala validada por Atienza, Pons, Balaguer y García-Mérita (2000) de la versión original Satisfaction With Life Scale (SWLS) (Diener, Emmons, Larsen \& Griffin, 1985). Este instrumento presenta cinco ítems precedidos por la frase "Dinos tu grado de desacuerdo o acuerdo en relación a las siguientes afirmaciones". Un ejemplo de ítem es "Si pudiera vivir mi vida otra vez, la repetiría tal y como ha sido". Las respuestas se recogerán mediante una escala tipo Likert que oscila entre 1 (muy en desacuerdo) y 5 (muy de acuerdo).

Satisfacción con la escuela (SATD). Para medir la diversión y el aburrimiento en la escuela, se utilizará el Cuestionario de Satisfacción con la Escuela validado por Castillo, Balaguer y Duda (2001) del Intrinsic Satisfaction Classroom Scale (ISC) (Duda \& Nicholls, 1992; Nicholls, 1989). Este instrumento presenta ocho ítems que miden el grado de satisfacción con la escuela en dos factores, tres ítems que miden el aburrimiento y cinco ítems que miden la diversión. La escala está precedida por la frase "Dinos tu grado de desacuerdo o acuerdo en relación a las siguientes afirmaciones, referidas a todas tus clases en el instituto". Un ejemplo de ítem del factor de diversión es "En la escuela normalmente encuentro que el tiempo vuela". Un ejemplo de ítem de aburrimiento es "En la escuela a 
menudo sueño despierto en lugar de pensar en las tareas de clase”. Las respuestas se recogerán mediante una escala tipo Likert que oscila entre 1 (muy en desacuerdo) y 5 (muy de acuerdo).

Satisfacción Intrínseca con las Materias (SIM). Para medir la satisfacción con las materias, se utilizará la versión adaptada a EF por Baena-Extremera, Granero-Gallegos, Bracho-Amador, y PérezQuero (2012) y a Matemáticas y Lengua por Baños, Baena-Extremera y Ortiz-Camacho (en prensa), de la versión original Sport Satisfaction In-strument (SSI) de Duda y Nicholls (1992). El SIM consta de 8 ítems para medir la satisfacción intrínseca mediante dos factores que miden satisfacción/diversión (5 ítems) y aburrimiento (3 ítems). La escala está precedida por la frase "Según la asignatura que doy en clase...", haciendo referencia a las materias de EF, Matemáticas y Lengua. Un ejemplo de ítem de satisfacción/diversión es "Normalmente me divierto en las clases de..." y, de aburrimiento es "En las clases de... a menudo me aburro". Las respuestas se recogerán en una escala de tipo Likert de 5 puntos que oscila desde 1 (muy en desacuerdo) a 5 (muy de acuerdo).

Niveles de actividad física en el tiempo libre. Para conocer los niveles de actividad física de los alumnos, se usarán cinco preguntas en un índice de cantidad de actividad físico-deportiva (Raitakari et al., 1994, Telama et al., 2005). Los ítems miden la frecuencia, duración e intensidad del ejercicio físico durante el tiempo libre y la participación en deportes organizados y competiciones deportivas. Los resultados más altos indican que los individuos son activos y los más bajos, que son más sedentarios.

\section{Procedimiento}

Parte del procedimiento de esta investigación ya ha sido llevada a cabo. Se contactó con los órganos competentes para poder llevar a cabo esta investigación, encontrando una respuesta afirmativa de participación en los estados de Nuevo León y Baja California (México) y la CCAA de Andalucía (España), obteniendo una respuesta negativa a participar en el estudio de la CCAA de Castilla y León (España). Posteriormente se aleatorizaron los centros de educación secundaria que participarían en el estudio, asistiendo con los directores de dichos centros para la solicitud de permiso. Los centros que dieron una respuesta negativa en la participación del estudio, se volvió a aleatorizar la selección de otro centro educativo hasta completar la muestra representativa. Los instrumentos para medir las variables se dividirán en dos, primero dispondrán de una hora para completar el Test de Matrices Progresivas de Raven, una vez lo finalicen dispondrán de 20-40 minutos para completar el cuestionario ad hoc con el resto de variables, informándoles que no habrá respuestas correctas o incorrectas en la segunda parte, por lo tanto, que contesten con la máxima sinceridad y honestidad. Los investigadores estarán en las aulas donde se realice la investigación por si alguno de los adolescentes tuviese alguna duda. Los estudiantes fueron informados del propósito del estudio y de sus derechos como participantes en el mismo, en base a la Declaración de Helsinki (2008).

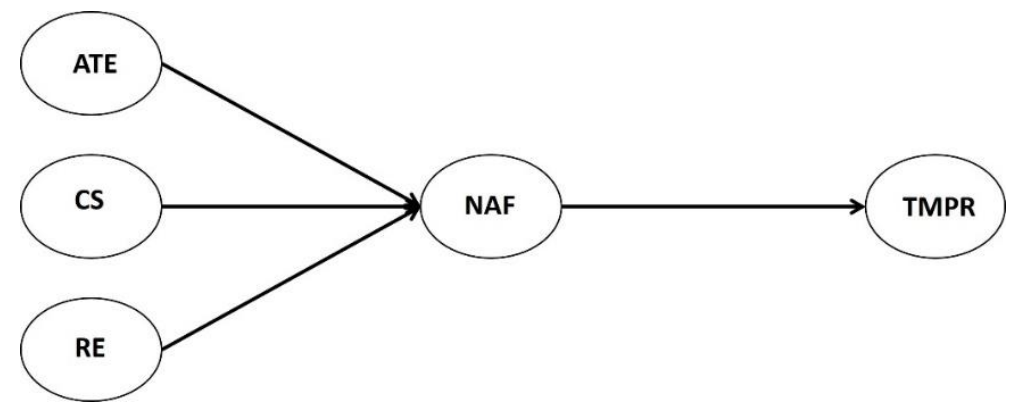

Figura 1. Posibles modelos a testear. Abreviaturas de las diferentes subescalas: ATE = atención emocional; CS = claridad de sentimientos; RE = reparación emocional; NAF = niveles de actividad física en el tiempo libre.

\section{Descripción de algunos de los modelos planteados}

Las posibilidades de investigación con estas variables podrían ser inmensa, pero es necesario delimitar y evaluar diferentes diseños de modelos de ecuaciones estructurales, acotando en dos planteamientos distintos. El primer planteamiento (ver Figura 1), se intentará dar respuesta a la influencia de la inteligencia emocional a través de los niveles de actividad física en su tiempo libre, 
sobre el coeficiente intelectual de los estudiantes. También se estudiará cómo se predice la satisfacción con la escuela desde los comportamientos negativos en el aula de EF y éste a su vez, desde la autoestima de los adolescentes (ver Figura 2).

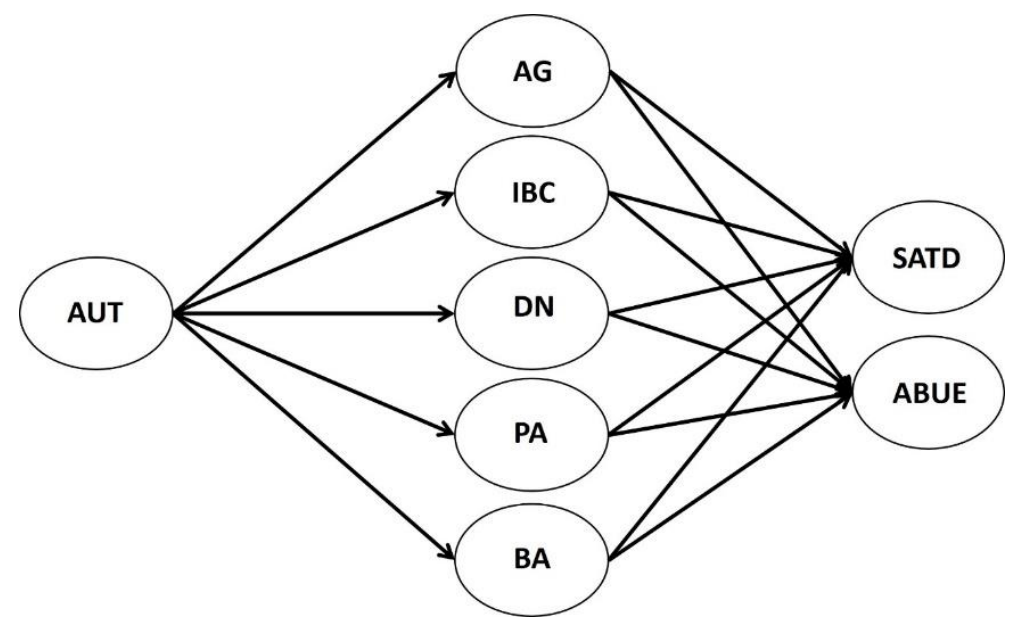

Figura 2. Posibles modelos a testear. Abreviaturas de las diferentes subescalas: AUT = autoestima; $A G=$ agresividad; IBC = irresponsabilidad y bajo compromiso; DN = desobediencia a las normas, PA = perturbar el ambiente de clase; $\mathrm{BA}=$ bajo autocontrol personal, $\mathrm{SATD}=$ satisfacción / diversión; ABUE = aburrimiento.

\section{Análisis estadísticos}

Para llevar a cabo este trabajo científico, el planteamiento de análisis estadístico debe pasar por lo siguiente.

En el objetivo general se realizará un análisis descriptivo de todos los factores que componen los instrumentos, calculando media, desviación típica, prueba de normalidad, índices de asimetría y fiabilidad de los factores, para cada uno de los estados que participaran en el estudio en función del sexo. Se analizará las diferencias que existan entre estados y países en función del sexo de los adolescentes.

Posteriormente, se analizará la estructura factorial de todos los instrumentos en cada estado aplicado y en función del sexo, mediante un análisis factorial confirmatorio (AFC) con el software LISREL; se examinará la consistencia interna mediante alfa de Cronbach, coeficiente de fiabilidad compuesta y varianza media extractada (AVE).

Respecto al primer objetivo, se realizará un AFC de la escala SIM para las materias de EF, Matemáticas y Lengua, así como de la escala SATV. Se calcularán varios índices de fiabilidad y de validez para cada instrumento (alfa de Cronbach, fiabilidad compuesta y AVE). A continuación, se realizará un análisis de conglomerados (cluster). Esta técnica multivariante busca agrupar variables tratando de lograr la máxima homogeneidad en cada grupo y las mayores diferencias entre ellos. Se realizará un análisis cluster jerárquico exploratorio con el método de Ward y a continuación, un segundo análisis cluster a través del método k-medias, utilizando las medias de los clusters obtenidos como el centro inicial de cada cluster. Posteriormente se comprobará la validez predictiva de los cluster mediante análisis univariante y para finalizar, se realizará un análisis residual para examinar el potencial de las diferencias entre los grupos de IMC y de rendimiento académico.

En relación al segundo objetivo, se analizará las propiedades psicométricas de Los instrumentos. Se llevará a cabo un AFC de la escala niveles de actividad física en el tiempo libre, del TMMS-24 y del Test de Matrices Progresivas del Raven. Se estudiarán varios índices de fiabilidad y validez, como el alfa de Cronbach, la fiabilidad compuesta, prueba de normalidad, índices de asimetría y la AVE para cada instrumento. En función de los resultados de la prueba de normalidad, se realizará un análisis de correlación entre las escalas. Para finalizar, se llevará a cabo diversos modelos de ecuaciones estructurales, para responder al objetivo tres de este trabajo. Los cálculos se realizarán con el software LISREL 8.80 y el paquete estadístico SPSS v.22. 
Protocolo de estudio de las variables académicas, psicológicas y de actividad física que influyen en el rendimiento académico de adolescentes mexicanos y españoles

Finalmente, el tercer objetivo se llevará a cabo igual que el segundo, se realizará un AFC para analizar la adecuación de los instrumentos (EAG, PECI y SATD), en los tres estados de los dos países estudiados, a continuación, se examinará los cálculos de índices de fiabilidad (alfa de Cronbach y fiabilidad compuesta) y de validez (AVE) para cada uno de los instrumentos. Posteriormente, se llevará a cabo un análisis de correlación entre los instrumentos utilizados, en función de los resultados de la prueba de normalidad. A continuación, realizarán diversos modelos de ecuaciones estructurales entre los factores para responder al objetivo. Los cálculos se realizarán con el software LISREL 8.80 y el paquete estadístico SPSS v.22.

\section{Resultados y discusión}

El objetivo fundamental de esta investigación es analizar las diferencias académicas, personales y ambientales de los estudiantes pertenecientes a los estados de Baja California y Nuevo León (México) y de la Comunidad Autónoma (CCAA) de Andalucía (España). El llevar a cabo un proyecto de investigación de esta envergadura, supondrá obtener gran cantidad información sobre los contextos educativos de diferentes estados y países. Se obtendrán resultados de gran relevancia sobre los factores que afectan al rendimiento académico de los estudiantes. Al adaptar y aplicar el trabajo a diferentes países, se espera analizar las diferencias entre los resultados obtenidos, para analizar qué variables y cómo afectan al rendimiento académico de los adolescentes. De esta forma, se podrá comprender mejor cómo dos países del mismo idioma, obtienen puntuaciones tan dispares en las evaluaciones del Informe Pisa.

El conocer el cómo se sienten de satisfechos los estudiantes con las materias que reciben y la relación de cada una de ellas con el rendimiento académico, servirá para analizar el pobre resultado obtenido por los adolescentes mexicanos en las evaluaciones del Informe Pisa (2015). Esto es importante ya que el promedio obtenido en dicha evaluación ha sido de 416 puntos en ciencias, 423 puntos en lectura y 408 puntos en matemáticas, muy alejado del promedio de la OCDE de 493, 493 y 490 puntos respectivamente. Recientemente se ha demostrado que la satisfacción con las materias predice la satisfacción con la escuela, y ésta a su vez, el rendimiento académico con adolescentes españoles (Baños et al., 2019), influyendo también en la satisfacción con la vida (Scharenberg, 2016). Por el contrario, un estudiante que se aburre en la escuela, predice el estrés y la ansiedad (Hui \& Sun, 2010), la depresión (Takakura, Wake \& Kobayashi, 2010), y aumenta la probabilidad de absentismo y abandono escolar (Delfabbro, Winefield, Winefield, Malvaso, \& Plueckhahn, 2017). Conocer la satisfacción con las materias de Matemáticas y español de los estudiantes mexicanos y españoles, puede ayudar a comprender los resultados tan bajos obtenidos por los mexicanos en las evaluaciones de matemáticas y lectura, tratando de explicar también los bajos niveles de bienestar subjetivo de los adolescentes mexicanos (OCDE, 2016).

En relación a la inteligencia emocional, los resultados del presente trabajo podrían revelar información interesante sobre cómo se relaciona esta variable, con los niveles de actividad física en el tiempo libre y el coeficiente intelectual de los adolescentes, analizando si existen diferencias entre los estudiantes de distintos países. En esta línea, despierta gran interés averiguar cómo la actividad física puede influir en una mayor salud emocional, un mejor funcionamiento cognitivo, autocontrol personal y una mejor calidad de vida (Asare \& Danquah, 2015; Brosnahan, Steffen, Lytle, Patterson \& Boostrom, 2004; Herazo-Beltrán et al., 2019). También estudiar cómo influye la actividad física en el coeficiente intelectual de los estudiantes nos puede revelar información de gran relevancia, ya que existen indicios que un IMC bajo, con unos niveles de actividad física altos, mejoran el rendimiento cognitivo de los adolescentes (Emerson \& Robertson, 2010; Goldberg et al., 2014; Parisi et al., 2010; Yu, Han, Cao, \& Guo, 2010).

Para finalizar, con este trabajo esperamos revelar resultados que ayuden a los docentes en el conocimiento de cómo manejar efectivamente los problemas de disciplina en el aula (Evertson \& Weinstein, 2006; Tsouloupas, Carson, Matthews, Grawitch, \& Barber, 2011), a través del dominio de las estrategias pedagógicas (Voss, Kunter \& Baumert, 2011) y el aprendizaje sobre técnicas de gestión del aula (Emmer \& Stough, 2001). De esta forma, en función de cómo el docente gestione y organice 
las sesiones, podrá influir en los comportamientos disruptivos de los estudiantes (Baños et al., 2019), y esto a su vez en la autoestima de los adolescentes (Baena-Extremera \& Granero-Gallegos, 2013; Góngora, \& Casullo, 2009; Lewis et al., 2013). Los resultados del presente trabajo, pueden llegar a aportar información de gran interés para la comunidad educativa en general, y en especial, para el sistema educativo español y mexicano.

\section{Referencias}

Adkins, D. L., Boychuk, J., Remple, M. S., \& Kleim, J. A. (2006). Motor training induces experience-specific patterns of plasticity across motor cortex and spinal cord. Journal of Applied Physiology, 101(6), 17761782. doi:10.1152/japplphysiol.00515.2006

Asare, M., \& Danquah, S. A. (2015). The relationship between physical activity, sedentary behaviour and mental health in Ghanaian adolescents. Child and Adolescent Psychiatry and Mental Health, 9(11), 1-8. doi:10.1186/s13034-015-0043-x

Atienza, F. L., Moreno, Y., \& Balaguer, I. (2000). Análisis de la dimensionalidad de la Escala de Autoestima de Rosenberg en una muestra de adolescentes valencianos. Revista de Psicología, 22, 29-42.

Atienza, F. L., Pons, D., Balaguer, I., \& García-Merita, M. (2000). Propiedades psicométricas de la Escala de Satisfacción con la Vida en adolescentes. Psicothema, 12(2), 314-319. doi:10.1016/S01918869(02)00332-X;

Baena-Extremera, A., \& Granero-Gallegos, A. (2013). Effects of an Adventure Education program in orientation towards learning, satisfaction and self-concept in secondary school. Revista Iberoamericana de Diagnóstico y Evaluacion-e Avaliacao Psicologica, 2(36), 163-182.

Baena-Extremera, A., Granero-Gallegos, A., Bracho-Amador, C., \& Pérez-Quero, F. J. (2012). Versión española del Sport Satisfaction Instrument (SSI) adaptado a la educación física. Revista de Psicodidáctica, 17(2), 377-396. doi: 10.1387/Rev.Psicodidact.4037

Baños, R., Baena-Extremera, A., \& Ortiz-Camacho, M. M. (en prensa). Prediction Model of Academic Performance and Satisfaction With School According to Some Subjects of Compulsory Secondary Education. Psychological Reports, 0033294118805004. doi: 10.1177\%2F0033294118805004

Baños, R. F., Baena-Extremera, A., Ortiz-Camacho, M. M., Zamarripa, J., Beltrán, A., \& Juvera-Portilla, J. L. (2019). Influencia de las competencias del profesorado de secundaria en los comportamientos disruptivos en el aula. Espiral. Cuadernos del Profesorado, 12(24), 3-10. doi: 10.25115/ecp.v12i24.21

Baños, R., Ortiz-Camacho, M. M., Baena-Extremera, A., \& Tristán-Rodríguez, J. L. (2017). Satisfaction, motivation and academic performance in students of secondary and high school: background, design, methodology and proposal of analysis for a research paper. Espiral. Cuadernos del profesorado, 10(20), 40-50. doi: 10.25115/ ecp.v10i20.1011

Brosnahan, J., Steffen, L. M., Lytle, L., Patterson, J., \& Boostrom, A. (2004). The relation between physical activity and mental health among Hispanic and non-Hispanic white adolescents. Archives of Pediatrics and Adolescent Medicine, 158, 818-823. doi: 10.1001/archpedi.158.8.818

Castillo, I., Balaguer, I., \& Duda, J. L. (2001). Perspectivas de meta de los adolescentes en el contexto académico. Psicothema, 13(1), 79-86.

Collins, T. A., Cook, C. R., Dart, E. H., Socie, D. G., Renshaw, T. L., \& Long, A. C. (2016). Improving classroom engagement among high school students with disruptive behavior: Evaluation of the class pass intervention. Psychology in the Schools, 53(2), 204-219. doi: 10.1002/pits.21893

Delfabbro, P., Winefield, H., Winefield, A., Malvaso, C., \& Plueckhahn, T. (2017). Factors Associated With Attrition in a 10-year Longitudinal Study of Young People: Implications for Studies of Employment in School Leavers. Australian Psychologist, 52(1), 41-51. doi: 10.1111/ap.12207

Diaz, K. M., \& Caso, J. (2018). Personal, school and family variables that predict the academic performance in spanish of mexican adolescents. Revista Mexicana de Psicologia, 35(2), 141-157.

Diener, E., Emmons, R., Larsen, R. J., \& Griffin, S. (1985). The Satisfaction With Life Scale. Journal of Personality Assessment, 49, 71-75. doi: 10.1207/s15327752jpa4901_13

Duda, J. L. \& Nicholls, J. G. (1992). Dimensions of achievement motivation in schoolwork and sport. Journal of Educational Psychology, 84(3), 290-299.

Emerson, E., \& Robertson, J. (2010). Obesity in young children with intellectual disabilities or borderline intellectual functioning. International Journal of Pediatric Obesity, 5(4), 320-326. 
Protocolo de estudio de las variables académicas, psicológicas y de actividad física que influyen en el rendimiento académico de adolescentes mexicanos y españoles

Emmer, E. T., \& Stough, L. M. (2001). Classroom management: A critical part of educational psychology, with implications for teacher education. Educational Psychologist, 36(2), 103-112. doi: 10.1207/S15326985EP3602_5.

Evertson, C. M., \& C. S. Weinstein. "Handbook of Classroom Management: Research." Practice and Contemporary Issues. Mahwah NJ (2006).

Feinberg, M. E., Sakuma, K. L., Hostetler, M., \& McHale, S. M. (2013). Enhancing sibling relationships to prevent adolescent problem behaviors: Theory, design and feasibility of Siblings Are Special. Evaluation and Program Planning, 36(1), 97-106. doi: 10.1016/j.evalprogplan.2012.08.003

Goldberg, S., Werbeloff, N., Fruchter, E., Portuguese, S., Davidson, M., \& Weiser, M. (2014). IQ and obesity in adolescence: a population-based, cross-sectional study. Pediatric Obesity, 9(6), 419-426. doi: 10.1111/j.2047-6310.2013.00203.x

Gómez-Veiga, I., Vila Chaves, J. O., Duque, G., \& García Madruga, J. A. (2018). A New Look to a Classic Issue: Reasoning and Academic Achievement at Secondary School. Frontiers in Psychology, 9, 400-412. doi: 10.3389/fpsyg.2018.00400

Góngora, V. C., \& Calluso, M. M. (2009). Validación de la escala de autoestima de Rosenberg en población general y en población clínica de la Ciudad de Buenos Aires. Revista Iberoamericana de Diagnóstico y Evaluación Psicológica, 27(1), 179-194.

González, J., Cayuela, D., \& López, C. (2019). Prosociality, physical educaction and emotional intelligence in school. Journal of Sport and Health Research, 11(1), 17-32.

Granero-Gallegos, A. \& Baena-Extremera, A. (2016). Validación española de la versión corta del Physical Education Classroom Instrument para la medición de conductas disruptivas en alumnado de secundaria. Cuadernos de Psicología del Deporte, 16(2), 89-98.

Herazo-Beltrán, Y., Campo-Ternera, L., García-Puello, F., Méndez, O., Suarez-Villa, M., Vásquez-De la Hoz, F., \& Núñez-Bravo, N. (2019). Relationship between Physical Activity and Emotional Intelligence and Bullying Among School Children. Revista de Psicología del Deporte, 28(1) 281-297.

Hui, E. K. P., \& Sun, R. C. F. (2010). Chinese children's perceived school satisfaction: The role of contextual and intrapersonal factors. Educational Psychology: An International Journal of Experimental Educational Psychology, 30(2), 155-172, doi: 10.1080/01443410903494452

Informe PISA (2015). Organización para la Cooperación y el Desarrollo Económicos.

Kulinna P. H., Cothran D., \& Regualos R. (2006) Teachers' reports of student misbehavior in physical education. Research Quarterly for Exercise and Sport, 77, 32-40. doi: 10.1080/02701367.2006.10599329

Lewis, K. M., Schure, M. B., Bavarian, N., DuBois, D. L., Day, J., Ji, P., .. \& Flay, B. R. (2013). Problem behavior and urban, low-income youth: A randomized controlled trial of Positive Action in Chicago. American Journal of Preventive Medicine, 44(6), 622-630. doi: 10.1016/j.amepre.2013.01.030

Mayer, J. D., \& Salovey, P. (1997). What is emotional intelligence? In P. Salovey \& D. Sluyter (Eds.), Emotional development and emotional intelligence: Implications for educators (pp. 3-31). New York: Basic Books.

Muhonen, H., Pakarinen, E., Poikkeus, A. M., Lerkkanen, M. K., \& Rasku-Puttonen, H. (2018). Quality of educational dialogue and association with students' academic performance. Learning and Instruction, 55, 67-79. doi: 10.1016/j.learninstruc.2017.09.007

Newberry, A.L. \& Duncan R.D. (2001). Roles of boredom and lifegoals in juveniledelinquency. Journal of Applied Social Psychology, 31, 527-541. doi: 10.1111/j.1559-1816.2001.tb02054.x

Nicholls, J. G. (1989). The competitive ethos and democratic education. Cambridge, MA: Harvard University Press.

OECD. (2016). Education at a Glance 2016: OECD Indicators. Paris: OECD Publishing.

Parisi, P., Verrotti, A., Paolino, M. C., Miano, S., Urbano, A., Bernabucci, M., \& Villa, M. P. (2010). Cognitive profile, parental education and BMI in children: reflections on common neuroendrocrino biological roots.Journal of Pediatric Endocrinology and Metabolism,23(11), 1133-1141. doi: 10.1515/jpem.2010.178

Raitakari, O., Porkka, K., Taimela, S., Telama, R., Rasanen, L., \& Vikari, J. (1994). Effects of persistent physical activity and inactivity on coronary risk factors in children and young adults. American Journalof Epidemiology, 140, 195-205. doi: 10.1093/oxfordjournals.aje.a117239

Ramos, A. M., Lopez-Fernandez, V., \& Llamas-Salguero, F. (2017). A relationship among creativity, immediateand-logic memory and academic achievement in secondary school. Academia y virtualidad, 10(1), 123130. doi: $10.18359 /$ ravi.2674

Espiral. Cuadernos del Profesorado | ISSN 1988-7701 | 2019, 12(25), 89-99 
Raven, J. (2000). The Ravens Progressive Matrices: Change and stability over culture and time. Cognitive Psychology, 41, 1-48. doi: 10.1006/cogp.1999.0735

Raven, J.C. (1938). Ravens standard progressive matrices. London: HK Lewis.

Raven, J.C., Styles, I., \& Raven, M. (1998). Ravens progressive matrices: SPM plus test booklet. San Antonio, TX: Harcourt Assessment.

Rosenberg, M. (1965). Society and the adolescent self-image. Princeton, NJ: Princeton University Press. doi: $10.2307 / 2575639$

Ruiz-Juan, F., Baena-Extremera, A., \& Baños, R. (2017). Nivel de actividad deportiva en el tiempo libre desde las etapas de cambio y motivación en estudiantes de Costa Rica, México y España. Cuadernos de Psicología del Deporte, 17(2), 53-64.

Salguero, J. M., Fernandez-Berrocal, P., Balluerka, N., \& Aritzeta, A. (2010). Measuring perceived emotional intelligence in the adolescent population: Psychometric properties of the Trait Meta-Mood Scale. Social Behavior and Personality: an international journal, 38(9), 1197-1209. doi: 10.2224/sbp.2010.38.9.1197

Salovey, P., Mayer, J. D., Goldman, S. L., Turvey, C., \& Palfai, T. P. (1995). Emotional attention, clarity, and repair: Exploring emotional intelligence using the Trait Meta-Mood Scale. In J. W. Pennebaker (Ed.), Emotion, disclosure, and health (pp. 125-151). Washington: American Psychological Association. doi: $10.1037 / 10182-006$

Scharenberg, K. (2016). The interplay of social and ethnic classroom composition, tracking, and gender on students' school satisfaction. Journal of Cognitive Education and Psychology, 15(2), 320-346. doi: 10.1891/1945-8959.15.2.320

Takakura, M., Wake, N., \& Kobayashi, M. (2010). The contextual effect of school satisfaction on health-risk behaviors in Japanese high school students. Journal of School Health, 80(11), 544-551. doi: 10.1111/j.1746-1561.2010.00540.x

Telama, R., Yang X., Viikari J., Vãlimãki I., Wanne, O., \& RaitakariO. (2005). Physical Activity from Childhood to Adulthood. A 21-YearTracking Study. American Journal of Preventive Medicine, 28, 267-273. doi: 10.1016/j.amepre.2004.12.003

Tsouloupas, C. N., Carson, R. L., Matthews, R., Grawitch, M. J., \& Barber, L. K. (2011). Exploring the association between teachers' perceived student misbehaviour and emotional exhaustion: The importance of teacher efficacy beliefs and emotion regulation. Educational Psychology, 30(2), 173-189. doi: 10.1080/01443410903494460.

Umstattd, M., Bridges, C., Schmid, T., Hecht, A., \& Pollack, K. (2019). Systematic review of how Play Streets impact opportunities for active play, physical activity, neighbour hoods, and communities. BMC Public Health, 19(1), 335-352. doi: 10.1186/s12889-019-6609-4

Usán, P., \& Salavera, C. (2019). El rendimiento escolar, la inteligencia emocional y el engagement académico en una muestra de escolares. Electronic Journal of Research in Education Psychology, 17(47), 5-26. doi: 10.25115/ejrep.v17i47.1879

Voss, T., Kunter, M., \& Baumert, J. (2011). Assessing teacher candidates' general pedagogical/psychological knowledge: Test construction and validation. Journal of Educational Psychology, 103(4), 952-969. doi: $10.1037 / \mathrm{a} 0025125$

Wassenaar, T., Wheatley, C., Beale, N., Salvan, P., Meaney, A., Possee, J., ... \& Johansen-Berg, H. (2019). Effects of a programme of vigorous physical activity during secondary school physical education on academic performance, fitness, cognition, mental health and the brain of adolescents (Fit to Study): study protocol for a cluster-randomised trial. Trials, 20, 189-203. doi: 10.31219/osf.io/t32zc

Wegner, L., \& Flisher, A. J. (2009). Leisure boredom and adolescent risk behaviour: A systematic literatura review. Journal of Child and Adolescent Mental Health,21(1), 1-28. doi: 10.2989/JCAMH.2009.21.1.4.806

Yang, H., \& Yoh, T. (2005). The relationship between free-time boredom and aggressivebehavioraltendenciesamongcollegestudentswithdisabilities. American Journal of Recreation Therapy, 4, 11-16.

Yu, Z. B., Han, S. P., Cao, X. G., \& Guo, X. R. (2010). Intelligence in relation to obesity: a systematic review and meta-analysis. Obesity Reviews, 11(9), 656-670. doi: 10.1111/j.1467-789X.2009.00656.x 